\title{
Evaluation of Pyrosequencing ${ }^{\circledR}$ Technology for the Identification of Clinically Relevant Non-Dematiaceous Yeasts and Related Species
}

\author{
C.I. Montero ${ }^{1,{ }^{*}, \text { Y.R. Shea }}{ }^{1}$, P.A. Jones ${ }^{1}$, S.M. Harrington ${ }^{2}$, N.E Tooke ${ }^{3}$, F.G Witebsky ${ }^{1}$, and \\ P.R. Murray ${ }^{1}$ \\ 1 Microbiology Service, Department of Laboratory Medicine, Clinical Center, National Institutes of Health, \\ U.S. Department of Health and Human Services, 10 Center Drive, MSC 1508, Bethesda, MD 20892-1508, \\ USA \\ 2 Department of Clinical Microbiology, Albany Medical Center, 43 New Scotland Ave. Albany, NY 12208 \\ USA
}

3 Biotage AB, Kungsgatan 76, Uppsala, SE-753 18 Sweden

\begin{abstract}
Pyrosequencing was used to identify 133 isolates of clinically relevant non-dematiaceous yeasts. These included 97 ATCC strains (42 type strains), 7 UAMH strains, and 29 clinical isolates. Isolates belonged to the following genera: Candida (18 species), Trichosporon (10), Cryptococcus (7), Malassezia (3), Rhodotorula (2), Geotrichum (1), Blastoschizomyces (1) and Kodamaea (1). Amplicons were obtained of a hypervariable ITS region and analyzed using Pyrosequencing technology. The data were evaluated by a BLAST search against the GenBank database and correlated with data obtained by conventional cycle sequencing of the ITS1-5.8S-ITS2 region. Cycle sequencing identified (78.9\%) if the isolates to the species level. Pyrosequencing technology identified $69.1 \%$. In $90.1 \%$ of all the strains tested, identification results of both sequencing methods were identical. Most Candida isolates can be identified to the species level by pyrosequencing. Trichosporon species and some Cryptococcus cannot be differentiated at the species level. Pyrosequencing can be used for the reliable identification of most commonly isolated nondematiaceous yeasts, with a reduction of cost per identification compared to conventional sequencing.
\end{abstract}

\section{Keywords}

Yeast; Candida; Pyrosequencing; Identification; ITS

\section{Introduction}

The increase in the population of severely immunocompromised patients has been associated with an increase of invasive fungal infections [1]. These infections remain a significant cause of morbidity and mortality in patients receiving chemotherapy for cancer or organ transplantation [1,2]. Early detection of infection and identification of the etiologic agent

\footnotetext{
*Address inquiries to: Clemente I. Montero, Department of Laboratory Medicine, 10 Center Drive MSC 1508, Bethesda MD 20892-1508, Phone: 301-402-2144, Fax: 301-402-1886, monteroc@cc.nih.gov.
} 
permits the initiation of appropriate antifungal therapy, with reduced morbidity and increased possibility for survival $[2,3]$.

Currently, more than 300 fungal species have been recognized as potential human pathogens [4]. Although most episodes of invasive fungal infection are still caused by Candica albicans, the proportion of infections caused by non-C. albicans species has risen substantially [5]. The widespread use of antifungal agents may have contributed to a shift in species distribution via the emergence of inherently resistant species as significant pathogens $[3,5]$. The increasing importance of these isolates as causes of life-threatening invasive fungal infections in high-risk patients has important diagnostic implications for the clinical laboratory. Timely identification of this increasing diversity of pathogens may be hampered by the limitation of current diagnostic methods. Conventional identification of yeasts relies upon a combination of morphological and biochemical features using tests that may require relatively long periods of incubation, are labor intensive and/or require subjective interpretation. Popular biochemical identification panels such as API 20C AUX or VITEK ID-YST (BioMerieux, Durham N.C) may have limited databases resulting in misidentification of some isolates [69]. Therefore, molecular methods offer an attractive, accurate and rapid alternative for the identification of pathogenic yeast.

Several platforms have been evaluated for identification of yeasts in which the rRNA genes are frequently the selected target $[10,11]$. The availability of conserved and highly variable regions within the rRNA and its presence in multiple copies in fungal cells (Figure 1) allows high sensitivity as well as accurate molecular identification for most yeasts $[10,12,13]$.

Pyrosequencing ${ }^{\circledR}$ technology is a relatively inexpensive, easy to use method that employs coupled enzymatic reactions to determine the nucleotide composition of a target DNA during the polymerization reaction, transforming the concomitant release of pyrophosphate into visible light [14]. In comparison to other methods, Pyrosequencing technology is limited to short fragments but if the target is properly selected it may provide enough information for accurate identification of most clinically relevant yeasts. In this study the conventional cycle sequencing (Sanger) method was compared to Pyrosequencing ${ }^{\circledR}$ analysis for identification of yeasts associated with human disease.

\section{Materials and Methods}

\section{Reference strains and clinical isolates}

In the present study 133 isolates were used, Table 1 and 2 present the reference strains tested. Table 3 includes the clinical isolates tested. Lyophilized isolates, were cultured on Sabouraud plates and incubated at $30^{\circ} \mathrm{C}$ for $3-5$ days before genomic DNA extraction. For the remaining strains genomic DNA was extracted directly from growth on Sabouraud agar slants.

Biochemical assimilation tests were performed on some of the clinical isolates and reference strains that showed discrepant results between the ATCC identification and cycle sequencing results. All biochemical tests were performed using the API 20C AUX clinical yeast system, following the manufacturer's recommendations (BioMerieux Durham N.C.).

\section{Cycle Sequencing}

Fungal DNA was extracted using the UltraClean Microbial DNA Isolation kit (MoBio Laboratories, Solana Beach, CA) following manufacturer's recommendations for yeast genomic DNA extraction. Amplification of the ITS region (ITS1-5.8S-ITS2) was performed by using 1 pmol of the ITS1 (5'-TCCGTAGGTGAACCTGCGG-3') and ITS4 (5'TCCTCCGCTTATTGATATGC-3') primers [15], 0.1 ig of extracted DNA and ultrapure water to a final volume of 25 il with PuReTaq Ready-To-Go PCR beads (GE Healthcare, Fairfield, 
CT). The DNA was denatured at $95^{\circ} \mathrm{C}$ for $5 \mathrm{~min}$ and then subjected to 35 cycles of amplification $\left(95^{\circ} \mathrm{C}\right.$ for $1 \mathrm{~min}, 55^{\circ} \mathrm{C}$ for $1 \mathrm{~min}$, and $72^{\circ} \mathrm{C}$ for $1 \mathrm{~min}$ ), followed by a $10 \mathrm{~min}$ extension at $72^{\circ}$ C. The presence of PCR products was confirmed by gel electrophoresis. Purification of the PCR product was performed using a Microcon YM-100 centrifugation filter device (Millipore Corporation, Bedford, MA.). Amplicons were then sequenced using the ITS1 and ITS4 primers and the Big Dye Terminator v 1.1 cycle sequencing kit (PerkinElmer Applied Biosystems, Foster City, CA). Excess dye terminators were removed using CleanSeq magnetic particles according to the guidelines of the manufacturer (Agencourt, Beverly MA). Fluorescence-based sequence analysis of the extension products was performed with the ABI 3100 genetic analyzer (Applied Biosystems/Hitachi, Foster City, CA). The resulting sequences were assembled using Lasergene SeqMan II software (DNA Star, Inc., Madison, WI), and compared to GenBank nucleotide sequences by using the BLASTN program (NCBI, Bethesda, MD). Sequences were aligned using the CLUSTAL W algorithm and phylogenetic trees were constructed using the Lasergene MegAlign software (DNASTAR Inc., Madison, WI).

\section{Pyrosequencing analysis}

PCR was performed using PuReTaq Ready-To-Go PCR beads (GE Healthcare, Fairfield, CT) and primers included in the PyroMark ${ }^{\mathrm{TM}}$ Fungi ASR (Biotage, Charlottesville, VA). The PCR cycle included a $5 \mathrm{~min}$. initial denaturation and $35 \mathrm{cycles}\left(95^{\circ} \mathrm{C}\right.$ for $30 \mathrm{sec}, 58^{\circ} \mathrm{C}$ for $30 \mathrm{sec}$, and $72^{\circ} \mathrm{C}$ for $30 \mathrm{sec}$ ), followed by a $5 \mathrm{~min}$. extension at $72^{\circ} \mathrm{C}$. PCR products were then confirmed by gel electrophoresis. Biotinylated amplicons were purified using streptavidin coated sepharose beads (GE Healthcare, Fairfield, CT) and purification was performed using the Vacuum Prep Workstation and PSQ ${ }^{\circledR}$ Sample preparation kit following a standard protocol (Biotage, Charlottesville, VA), with annealing at a final sequencing primer concentration of $0.4 \mu \mathrm{M}$. Pyro Gold reagents were loaded into the PSQ reagent cartridge in a PyroMark ID unit (Biotage, Charlottesville, VA) for sequencing using 15 dispensation cycles (CTGA). The primer Seq2Pyro (5'-TAGTCTTGCGTGATGTGAGG-3'), not included in the PyroMark Fungi ASR, was used for sequence identification of $C$. catenulata and B. capitatus. Regardless of the quality of the Pyrogram ${ }^{\mathrm{TM}}$ assessed by the PyroMark ID software, all Pyrogram reports generated were submitted as BLAST queries through GenBank (NCBI, Bethesda, MD). One inherent limitation of the Pyrosequencing technology is the determination of the number of incorporated nucleotides in homopolymeric regions. A non-linear light output resulting from the sequential incorporation of more than five to six identical nucleotides may require adjustments of the software algorithms used for the integration of light intensities [16]. Therefore, visual interpretation of Pyrogram reports targeting these regions is recommended. Nonetheless, no "visual reading" adjustment was performed for any of the sequences obtained. Pyrogram reports that failed to provide an identifiable signal were annotated as "no signal" (Table 3).

\section{Results}

For all of the species successfully identified within our samples, sequencing of 20-30 bases of the selected ITS2 region (Figure 1) was sufficient for differentiation. A summary of the concordant and discordant identification results on all of the reference strains is presented in Table 1 and Table 2, respectively. Results of the identification of the clinical isolates included in this study are presented in Table 3. Cycle sequencing of two of the clinical isolates identified by biochemical methods as $C$. parapsilosis provided sequences homologous to $C$.

parapsilosis and C. orthopsilosis entries in GenBank. C. orthopsilosis is a newly defined species within the $C$. parapsilosis species complex [17]. However, differentiation within this species complex may be clinically irrelevant $[17,18]$. 
Of the 133 clinical isolates and reference strains used in this study (43 species), a total of 119 (38 species) resulted in interpretable Pyrogram reports. No signal was generated for 14 strains (6 species) and correspond to isolates of C. lambica, Geotrichum candidum, Kodamaea ohmeri, C. lipolytica, C. zeylanoides 20393, and C. rugosa ATCC 96275. With the exception of Geotrichum candidum, PCR amplicons were successfully generated for these 5 species without any apparent reduction in yield or additional bands.

Our results using Pyrosequencing technology are presented in Table 4 and include the first 30 base pairs of all clinical isolates or reference strains used in this study. A total of 54 sequences that represent 38 different species are presented. All of these species were identified at the genus level and 27 of them at the species level. Neither method targeting the ITS region was able to identify at the species level the remaining 11 species. These species consist of all Trichosporon species (except $T$. pullulans), all isolates from the $C$. neoformans species complex and some strains from the Cryptococcus luteolus/Bullera species group.

\section{Discussion}

In this work we present an evaluation of Pyrosequencing technology as a tool for the identification of the most commonly isolated non-dematiaceous yeasts $[5,19]$. The aim of the study was to compare the performance of this technology with the conventional cycle sequencing method. The targeted regions selected for these methods differ greatly in size, ITS1-5.8S-ITS2 (400-900bp) for cycle sequencing versus a variable region of size limited by the Pyrosequencing technical constraints ( $<60 \mathrm{bp}$ ). A previous study showed that conventional sequencing of the ITS2 region of 373 reference and clinical yeast isolates ( 86 species, mostly Candida species) allowed the identification $98.8 \%$ of the species tested [13]. Here, the resolving power of this hyper-variable ITS2 region is further evaluated by selecting a small 30 bp region for identification of yeasts by Pyrosequencing technology.

Previous reports have shown the utility of Pyrosequencing analysis for the identification of clinically relevant yeasts $[20,21]$. Those studies however, were limited to not more than 5 different species. In our study targeting a different region, we were able to extend this approach and identify 27 different yeast species to the species level and 11 more to the genus level (Tables 1,2 and 3). Taking into consideration recent reports on global trends of species distribution of invasive candidiasis [5,22]; we estimate that this test is potentially capable of identifying $95 \%$ of all Candida clinicalisolates to the species level. However, we found some limitations. Although our primer sets generate suitable PCR products from C. lipolytica, C. lambica, G. candidum, Kodamaea ohmeri, C. zeylanoides 20393, C. rugosa ATCC 96275, identification by the Pyrosequencing method was not possible. Problems of annealing between the primers used for sequencing and the target rDNA sequence of these strains may explain the absence of signal when Pyrosequencing analysis is performed.

As described previously, this assay is unable to identify to the species level members of the Cryptococcus neoformans species complex, Cryptococcus luteolus/Bullera species group, and most Trichosporon species previously grouped within Trichosporon beigelli [23]. The Cryptococcus neoformans species complex, is now considered to be conformed of two species, C. neoformans (serogroups A \& D) and Cryptococcus gattii (serogroups B and C) [24,25]. However the clinical relevance of this differentiation has not been firmly established [25,26]. If differentiation within the $C$. neoformans sp. complex is warranted, Pyrosequencing analysis or cycle sequencing of the rDNA will not adequately resolve these species unless sequencing of a "fast-evolving" region (such as the intergenic spacer region-IGS) is used for this purpose $[24,27]$. 
A similar problem was observed within Trichosporon, a group that has recently undergone an extensive taxonomic re-evaluation. Sequencing the ITS region distinguishes three clades [23]. As shown in Table 4, the Pyrosequencing results of species in Clade I ( T. jiroveci, T. moniliforme, and T. mucoides ) and Clade III (T. asahii, T. asteroides, T. coremiiforme, T. faecale, $\mathrm{T}$. ovoides and $\mathrm{T}$. inkin ) possesses unique sequence signatures [23]. Our strain collection did not include any member of Clade II (e.g. T. domesticum, T. montevideense). However, using bioinformatics methods and sequences publicly available in GenBank, we found that $T$. pullulans yielded a Pyrogram report that was distinguishable from all clades. However this species now renamed Guehomyces pullulans [28] should not be considered a significant infectious agent in humans [29].

The Cryptococcus laurentii species complex (15 species) can be divided into two phylogenetically distinct groups [30]. Based on the sequences determined by Takashima et al. [30], our assay can differentiate among all the species currently included withinphylogenetic group I (e.g. C. flavescens, $C$. aureus, C. laurentii). However, differences in the target annealing sequences of the sequencing primers (results not shown) prevents obtaining evaluable sequence data results from any member of phylogenetic group II (e.g. C. carnescens, C. peneaus, C. victoriae); These species do not appear to be clinically relevant and are primarily associated with marine or soil environments [30].

One of the unanticipated outcomes of our study is the finding of discrepancies between the species designations of some of the ATCC isolates and the biochemical and molecular identification obtained by the methods employed here (Table 2). These discrepancies are especially evident with regard to the identification of the reference strains of Cryptococcus luteolus. The type strain C. luteolus ATCC 32044 was identified by both sequencing methods as C. luteolus. However, all other "C. luteolus" strains (ATCC 42279, ATCC 42280, ATCC 44440) were not identified satisfactorily at the species level by either sequencing method (Table 3 ). It is unclear if these isolates belong to the species C. luteolus.

One of the technical limitations of Pyrosequencing technology is the accurate resolution of long homopolymers. Unfortunately, the region targeted in our assay included some of theses sequences. Examples of these homopolymers are shown for the sequences obtained from C. terreus, M. sympodialis, C. catenulata and C. neoformans (Table 4). The presence of homopolymers in the target region of $C$. neoformans increases the number of expected polymorphisms from two (according to cycle sequencing and GenBank databases) to five. Homopolymers are also present in the target sequence of Cryptococcus terreus, based on the current GenBank databases. Two polymorphisms are expected within this region. However, in two of the reference strains tested, the Pyrosequencing result obtained was different by one nucleotide deletion from either one of these polymorphisms.

It has been previously estimated that DNA polymerase in the presence of apyrase (the enzyme that eliminates excess deoxynucleotides during Pyrosequencing analysis), is capable of incorporating up to 10 identical adjacent nucleotides within a homopolymeric region [31]. However, in the experimental conditions employed, it seems that Pyrosequencing analysis may not reliably define the homopolymeric regions of more than 3 consecutive adenine bases in certain sequences (C. neoformans sequence, Table 4). However, adenine homopolymers may be resolved if they are located at the beginning of the region to be sequenced (C. catenulata sequence, Table 4). A condition that accentuates this problem is the excess of template (PCR product) that may enhance the non-synchronized extension of DNA fragments. Some references suggest that, upon re-sequencing, it is possible to use a dispensation cycle in which the problem homopolymeric nucleotide is added twice to ensure complete polymerization and reduction of these non-synchronized DNA fragments [16]. However, the utility of this approach has to be determined empirically based on the sequence of the most frequent clinical isolates. 
In four cases (Table 4, isolates with 93\% similarity), 2 unexpected nucleotide differences within the first 30 sequenced bases were observed in the following strains: $M$. sympodialis ATCC 96803, Cryptococcus gatti ATCC 32609, Cryptococcus neoformans ATCC 32045 and Cryptococcus neoformans ATCC 32268. In each case, BLASTN searches of the obtained sequences in GenBank resulted in correct identification with a $100 \%$ match. However, BLASTN obtained these results from portions of the submitted Pyrosequencing results excluding sections with problems of deletion or addition of bases within the homopolymeric regions.

It is important to emphasize that not all polymorphisms detected correspond to artifacts. In some instances, the intra-species variability observed was corroborated by conventional sequencing (e.g. C. haemulonii, C. parapsilosis, C. laurentii, C. luteolus, C. neoformans, $C$. terreus). Further optimization of the conditions tested for Pyrosequencing analysis (for instance, controlling the amount of template used per reaction) might reduce non-synchronized DNA polymerization associated with some of the sequence polymorphisms observed here.

The routine utilization of a molecular approach for the identification of clinically relevant yeasts will allow an accurate identification of the isolates and will provide a more reliable correlation of possible differences in clinical outcome virulence and epidemiology. The proposed identification approach provides an alternative tool for the differentiation between the closely related C. albicans and C. dubliniensis. C. dubliniensis was described as being more prevalent among immunocompromised and HIV infected populations [32].

In our experience, when compared to sequencing methods, the use of carbohydrate assimilation kits for identification of yeasts is less reliable. When compared to conventional cycle sequencing, the implementation of Pyrosequencing analysis in the molecular identification scheme for yeasts is associated with a reduction in processing time of at least 4 hours per batch. When compared to "in house" sequencing assays, reactive cost per reaction (including cost of PyroMark Fungi ASR kit) is reduced by $13 \%$. However, if the sample preparation buffers are prepared in the laboratory, the reactive cost per reaction is reduced by $46 \%$.

The present approach will allow the identification to the species level of the majority of clinical yeast isolates (>95\%). However, similar to the limitations of cycle sequencing of the ITS region, the Pyrosequencing assay evaluated here will not differentiate among species included within the Cryptococcus neoformans species complex and the Trichosporon species complex. Additionally, our Pyrosequencing approach was not able to obtain any sequence information for five species. However, these species may probably represent less than $1 \%$ of all clinical yeast isolates in the majority of clinical laboratories [5,22]. They account for not more than $0.06 \%$ of the clinical isolates identified in our institution since 2001 (data not shown).

The workflow, workload and financial resources available in each clinical laboratory will determine if DNA-based methods of identification could be used. Pyrosequencing technology represents a rapid and less expensive sequencing alternative that can be implemented in a clinical laboratory by technologists trained in conventional sequencing. The cost advantage of pyrosequencing is maximized when a high volume of samples is processed; therefore, standardization of targets and methodologies for the identification of different groups of bacteria, molds and yeasts will favor the decision to incorporate this technology into clinical laboratory settings.

The present study demonstrates an alternative and relatively inexpensive approach for sequencing-based identification of non-dematiaceous yeasts. A database of reference strains is provided (Table 4) that can be uploaded into the Pyrosequencing ${ }^{\circledR}$ identification software (IdentiFire ${ }^{\mathrm{TM}}$ Software) for the rapid implementation of this technology. The use of the database 
and the IdentiFire Software will allow the identification to the species or genus level of the vast majority of non-dematiaceous yeast of clinical significance.

\section{Acknowledgements}

We thank Patricia S. Conville, Department of Laboratory Medicine, Warren G. Magnuson Clinical Center, NIH, for her technical assistance. This research was supported by the Intramural Research Program of the NIH Clinical Center. The views expressed herein are those of the authors and should not be construed as those of the U.S. Department of Health and Human Services.

\section{References}

1. Walsh TJ, Groll A, Hiemenz J, Fleming R, Roilides E, Anaissie E. Infections due to emerging and uncommon medically important fungal pathogens. Clin Microbiol Infect 2004;10(Suppl 1):48-66. [PubMed: 14748802]

2. Vartivarian SE, Anaissie EJ, Bodey GP. Emerging fungal pathogens in immunocompromised patients: classification, diagnosis, and management. Clin Infect Dis 1993;17(Suppl 2):S487-S491. [PubMed: 8274615]

3. Aperis G, Myriounis N, Spanakis EK, Mylonakis E. Developments in the treatment of candidiasis: more choices and new challenges. Expert Opin Investig Drugs 2006;15:1319-1336.

4. Taylor LH, Latham SM, Woolhouse ME. Risk factors for human disease emergence. Philos Trans R Soc Lond B Biol Sci 2001;356:983-989. [PubMed: 11516376]

5. Pfaller MA, Diekema DJ. Epidemiology of invasive candidiasis: a persistent public health problem. Clin Microbiol Rev 2007;20:133-163. [PubMed: 17223626]

6. Bhally HS, Jain S, Shields C, Halsey N, Cristofalo E, Merz WG. Infection in a neonate caused by Pichia fabianii: importance of molecular identification. Med Mycol 2006;44:185-187. [PubMed: 16519022]

7. Ramani R, Gromadzki S, Pincus DH, Salkin IF, Chaturvedi V. Efficacy of API 20C and ID 32C systems for identification of common and rare clinical yeast isolates. J Clin Microbiol 1998;36:3396-3398. [PubMed: 9774605]

8. Coignard C, Hurst SF, Benjamin LE, Brandt ME, Warnock DW, Morrison CJ. Resolution of discrepant results for Candida species identification by using DNA probes. J Clin Microbiol 2004;42:858-861. [PubMed: 14766873]

9. Massonet C, Van Eldere J, Vaneechoutte M, De Baere T, Verhaegen J, Lagrou K. Comparison of VITEK 2 with ITS2-fragment length polymorphism analysis for identification of yeast species. J Clin Microbiol 2004;42:2209-2011. [PubMed: 15131191]

10. Chen SC, Halliday CL, Meyer W. A review of nucleic acid-based diagnostic tests for systemic mycoses with an emphasis on polymerase chain reaction-based assays. Med Mycol 2002;40:333357. [PubMed: 12230214]

11. Summerbell RC, et al. Microcoding: the second step in DNA barcoding. Philos Trans R Soc Lond B Biol Sci 2005;360:1897-1903. [PubMed: 16214747]

12. Linton CJ, et al. Molecular identification of unusual pathogenic yeast isolates by large ribosomal subunit gene sequencing: 2 years of experience at the United Kingdom mycology reference laboratory. J Clin Microbiol 2007;45:1152-1158. [PubMed: 17251397]

13. Leaw SN, Chang HC, Sun HF, Barton R, Bouchara JP, Chang TC. Identification of medically important yeast species by sequence analysis of the internal transcribed spacer regions. J Clin Microbiol 2006;44:693-699. [PubMed: 16517841]

14. Ahmadian A, Ehn M, Hober S. Pyrosequencing: history, biochemistry and future. Clin Chim Acta 2006;363:83-94. [PubMed: 16165119]

15. White, T.; Bruns, T.; Lee, S.; Taylor, J. Amplification and direct sequencing of fungal ribosomal RNA genes for phylogenetics. In: Innis, MA.; Gelfand, DH.; Sninsky, JJ.; White, TJ., editors. PCR protocols: a guide to methods and applications. Academic Press San Diego; California: 1990.

16. Fakhrai-Rad H, Pourmand N, Ronaghi M. Pyrosequencing: an accurate detection platform for single nucleotide polymorphisms. Hum Mutat 2002;19:479-485. [PubMed: 11968080] 
17. Tavanti A, Davidson AD, Gow NAR, Maiden MCJ, Odds FC. Candida orthopsilosis and Candida metapsilosis spp. nov to replace Candida parapsilosis groups II and III. Journal of Clinical Microbiology 2005;43:284-292. [PubMed: 15634984]

18. Gacser A, Schafer W, Nosanchuk J, Salomon S, Nosanchuk J. Virulence of Candida parapsilosis, $C$. orthopsilosis and C. metapsilosis in reconstituted human tissue models. Fungal Genetics. 2007Article in press

19. Hazen KC. New and emerging yeast pathogens. Clin Microbiol Rev 1995;8:462-478. [PubMed: 8665465]

20. Gharizadeh B, et al. Identification of medically important fungi by the Pyrosequencing technology. Mycoses 2004;47:29-33. [PubMed: 14998396]

21. Trama JP, Mordechai E, Adelson ME. Detection and identification of Candida species associated with Candida vaginitis by real-time PCR and pyrosequencing. Mol Cell Probes 2005;19:145-152. [PubMed: 15680216]

22. Pfaller MA, et al. Results from the ARTEMIS DISK Global Antifungal Surveillance study, 1997 to 2005: an 8.5-year analysis of susceptibilities of Candida species and other yeast species to fluconazole and voriconazole determined by CLSI standardized disk diffusion testing. J Clin Microbiol 2007;45:1735-1745. [PubMed: 17442797]

23. Rodriguez-Tudela JL, et al. Susceptibility patterns and molecular identification of Trichosporon species. Antimicrob Agents Chemother 2005;49:4026-4034. [PubMed: 16189076]

24. Diaz MR, Fell JW. Use of a suspension array for rapid identification of the varieties and genotypes of the Cryptococcus neoformans species complex. J Clin Microbiol 2005;43:3662-3672. [PubMed: 16081894]

25. Kwon-Chung KJ, Varma A. Do major species concepts support one, two or more species within Cryptococcus neoformans? FEMS Yeast Res 2006;6:574-587. [PubMed: 16696653]

26. Hazen, KC.; Howell, SA. Candida, Cryptococcus and other yeasts of medical importance. In: Murray, PR.; Baron, EJ.; Jorgensen, JH.; Landry, ML.; Pfaller, MA., editors. Manual of Clinical Microbiology. 9. ASM Press; Washington DC: 2007. p. 1762-1788.

27. Bovers M, et al. Identification of genotypically diverse Cryptococcus neoformans and Cryptococcus gattii isolates by Luminex xMAP technology. J Clin Microbiol 2007;45:1874-1883. [PubMed: 17442792]

28. Fell JW, Scorzetti G. Reassignment of the basidiomycetous yeasts Trichosporon pullulans to Guehomyces pullulans gen. nov., comb. nov. and Hyalodendron lignicola to Trichosporon lignicola comb. nov. Int J Syst Evol Microbiol 2004;54:995-998. [PubMed: 15143054]

29. Holland SM, Shea YR, Kwon-Chung J. Regarding Trichosporon pullulans infection in 2 patients with chronic granulomatous disease. Journal of Allergy and Clinical Immunology 2004;114:206.

30. Takashima M, Sugita T, Shinoda T, Nakase T. Three new combinations from the Cryptococcus laurentii complex: Cryptococcus aureus, Cryptococcus carnescens and Cryptococcus peneaus. International Journal of Systematic and Evolutionary Microbiology 2003;53:1187-1194. [PubMed: 12892148]

31. Ronaghi M. Improved performance of pyrosequencing using single-stranded DNA-binding protein. Anal Biochem 2000;286:282-288. [PubMed: 11067751]

32. Sullivan DJ, Moran GP, Coleman DC. Candida dubliniensis: ten years on. FEMS Microbiol Lett 2005;253:9-17. [PubMed: 16213674]

33. de Hoog, GS.; Guarro, J.; Gene, J.; Figueras, MJ. Atlas of Clinical Fungi. 2. Centraalbureau voor Schimmelcultures/Universitat Rovira i Virgili; Utrecht: 2000.

34. Giammanco GM, Melilli D, Pizzo G. Candida pararugosa isolation from the oral cavity of an Italian denture wearer. Res Microbiol 2004;155:571-574. [PubMed: 15313258]

35. Bai FY, Takashima M, Nakase T. Description of Bullera kunmingensis sp. nov., and clarification of the taxonomic status of Bullera sinensis and its synonyms based on molecular phylogenetic analysis. FEMS Yeast Res 2001;1:103-109. [PubMed: 12702355]

36. Gueho E, Midgley G, Guillot J. The genus Malassezia with description of four new species. Antonie Van Leeuwenhoek 1996;69:337-355. [PubMed: 8836432]

37. CBS. Centraalbureau vor Schimmelcultures. Fungal Biodiversity Center Yeast Database. 2007. http://www.cbs.knaw.nl/yeast/BioloMICS.aspx 


\section{$5.8 S$ rDNA (SSU)}

$156 \mathrm{bp}$

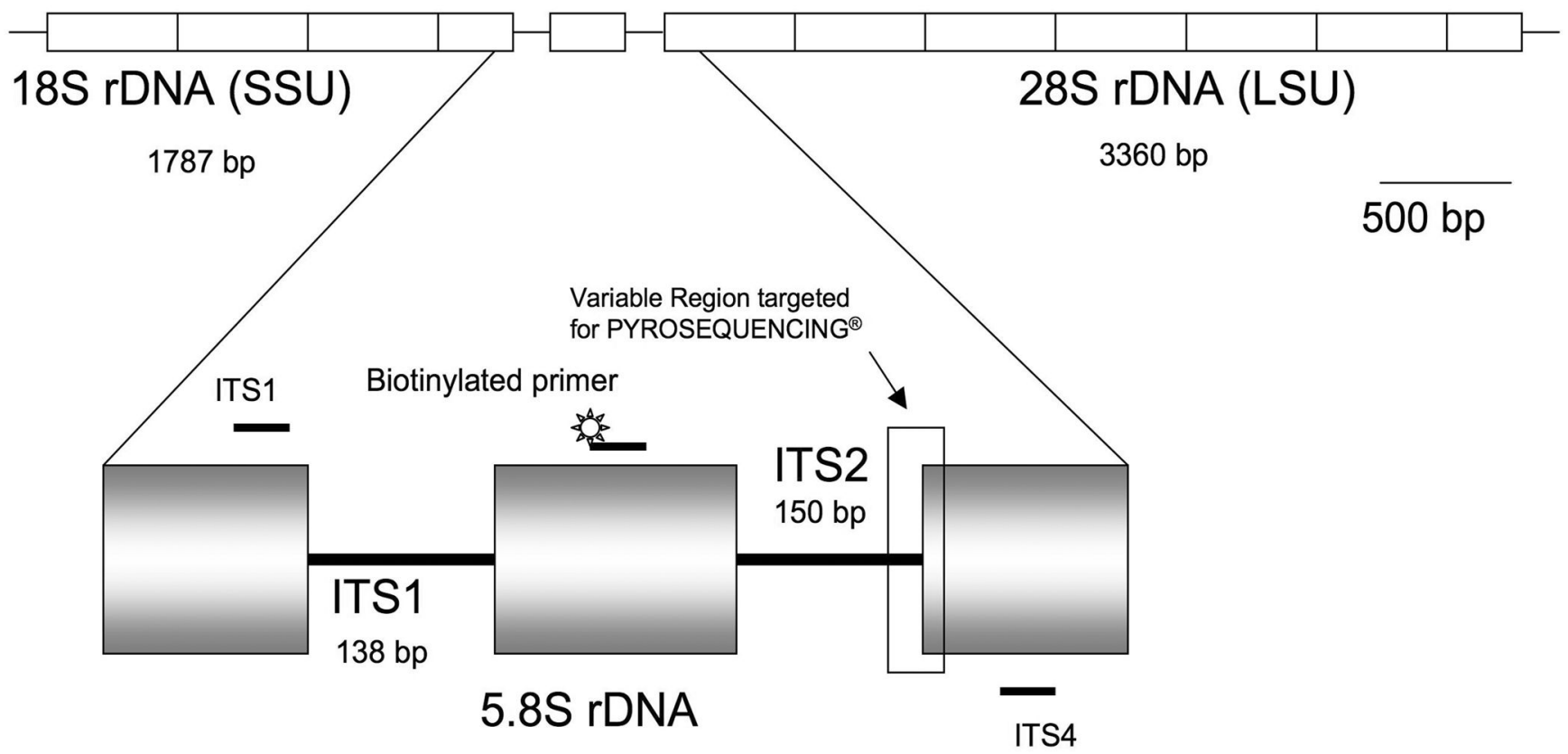

Figure 1. Diagram of the ribosomal DNA cluster of fungi and location of the primers used for this study

The ribosomal DNA (rDNA) gene is a tandam array of at least 50-100 copies in the haploid genome of all fungi (7). Diagram based on one single gene repeat in the genome sequence of C . albicans SC5314(7,20).(LSU)Large ribosomal subunit,(SSU) Small ribosomal subunit, Intergenic transcribed spacer region 1 (ITSI), Intergenic transcribed spacer region 2 (ITS2). Small bars (ITS1,ITS4) refers to approximate location of the universal fungal primers(38). 


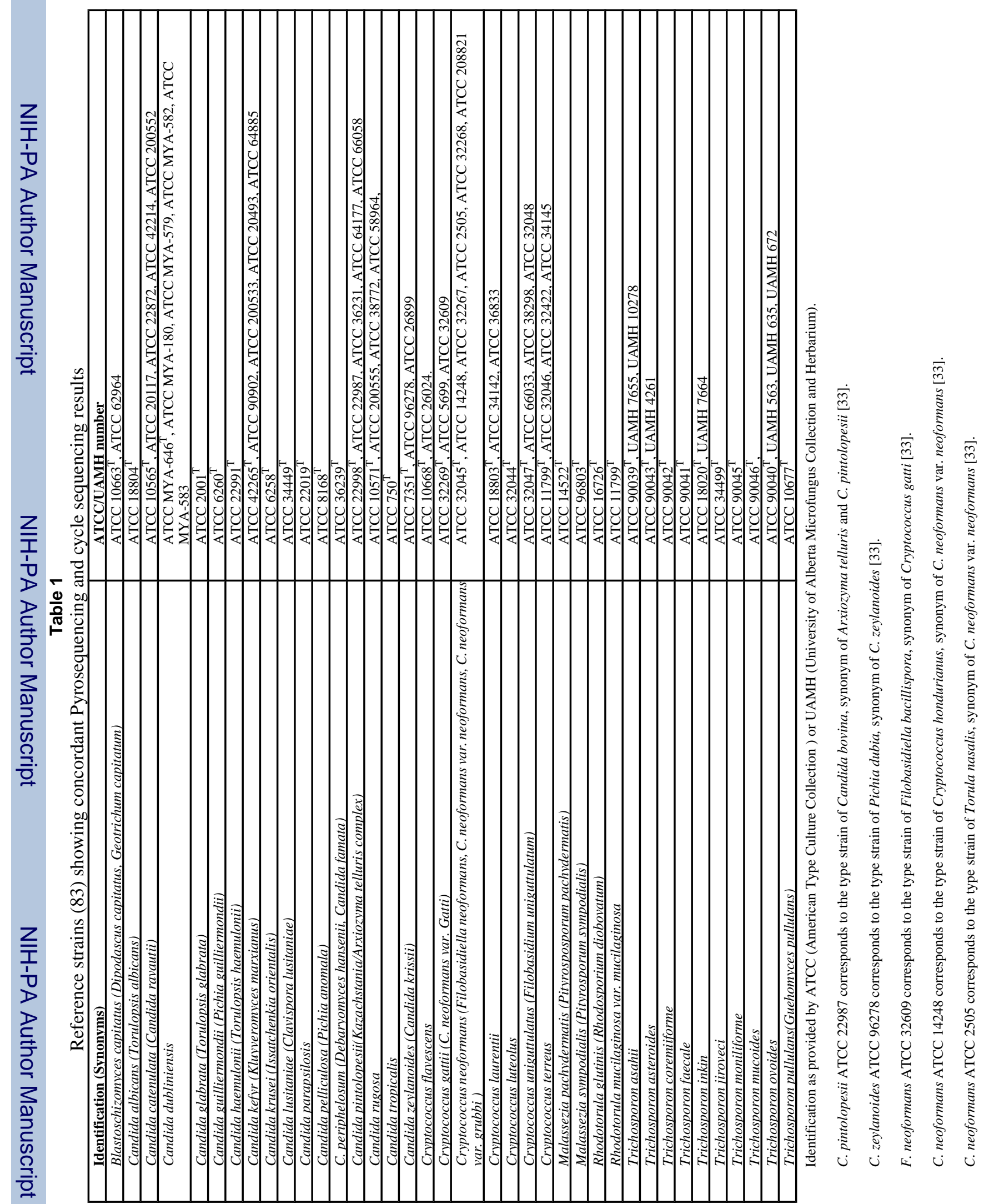




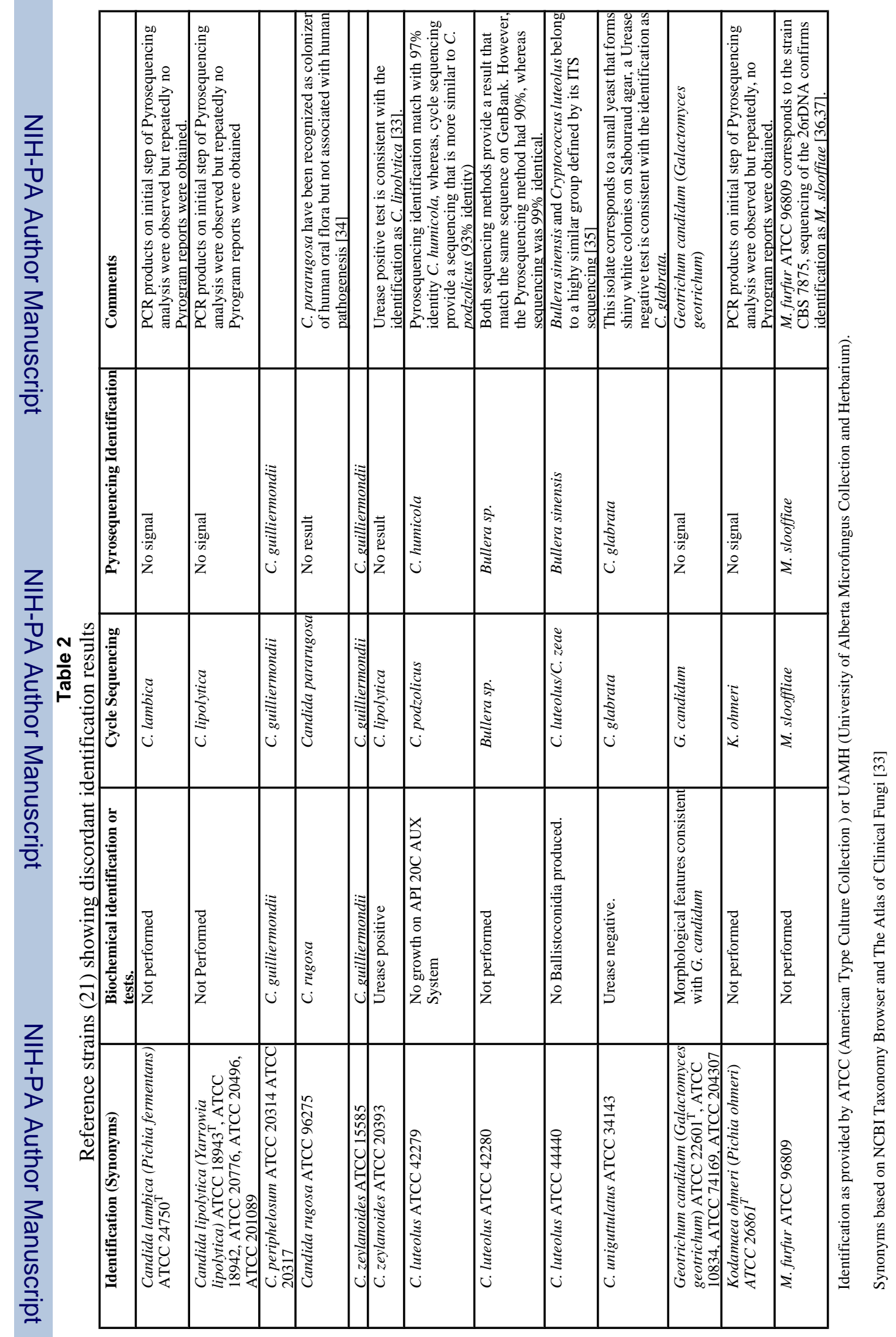




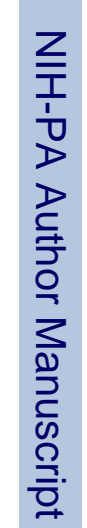

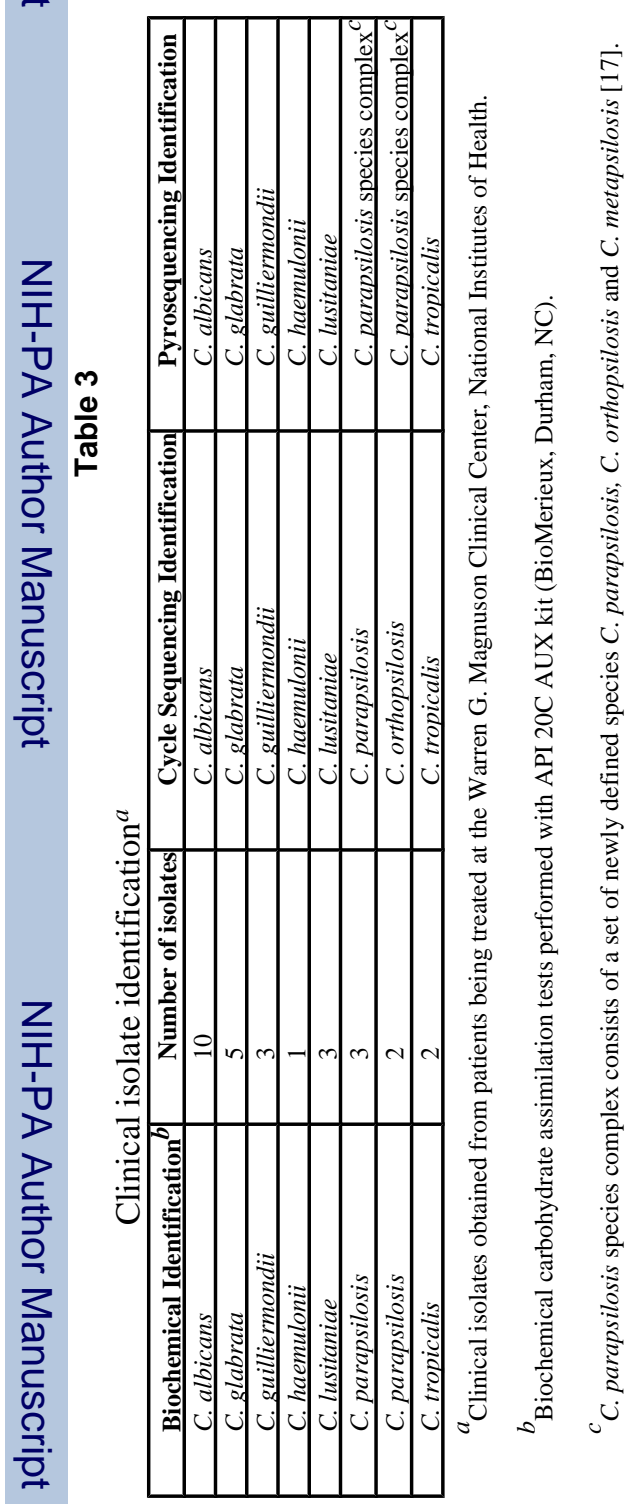




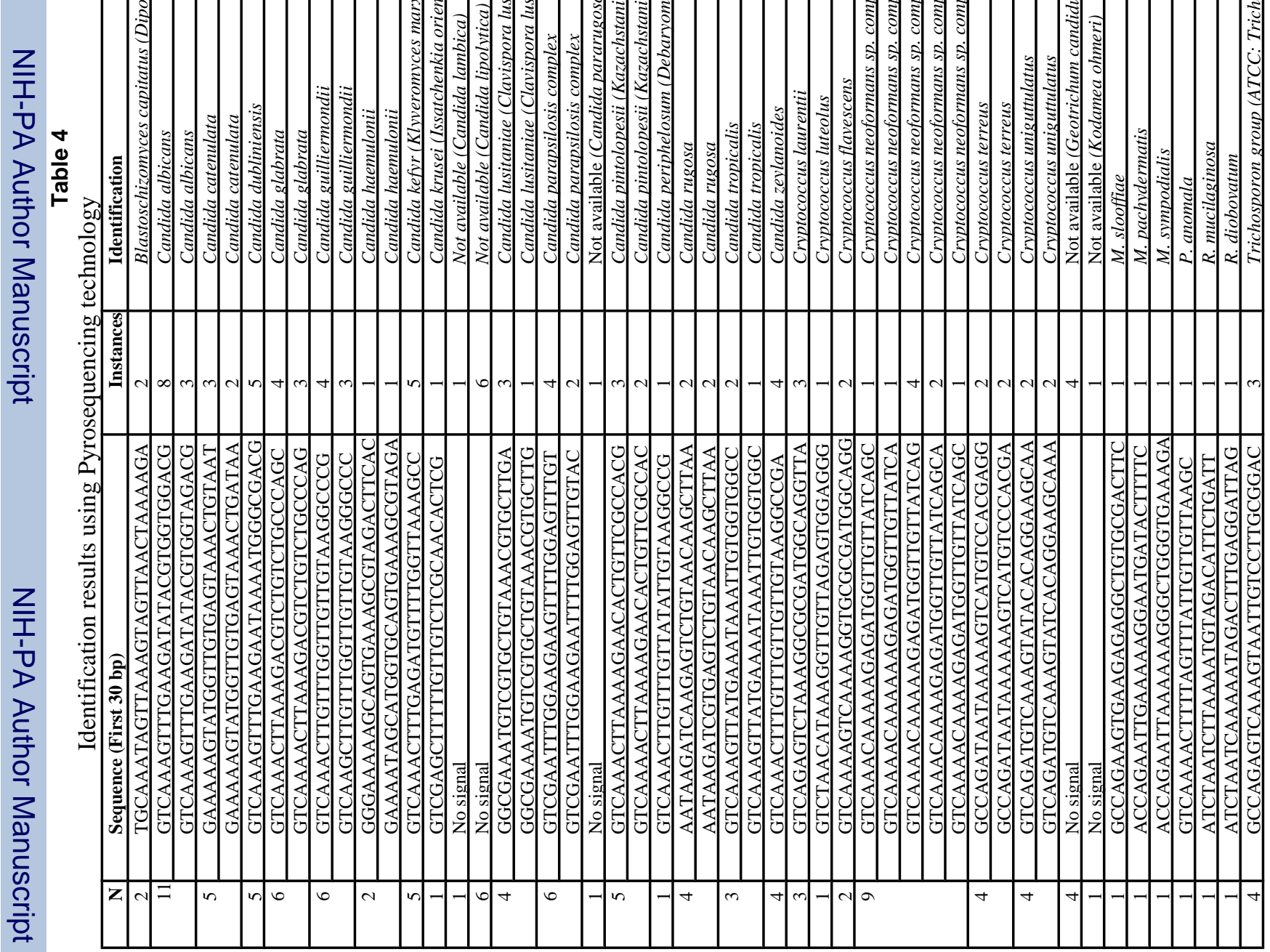




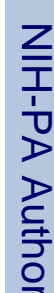

c

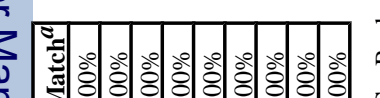

$7=-7=-=0$
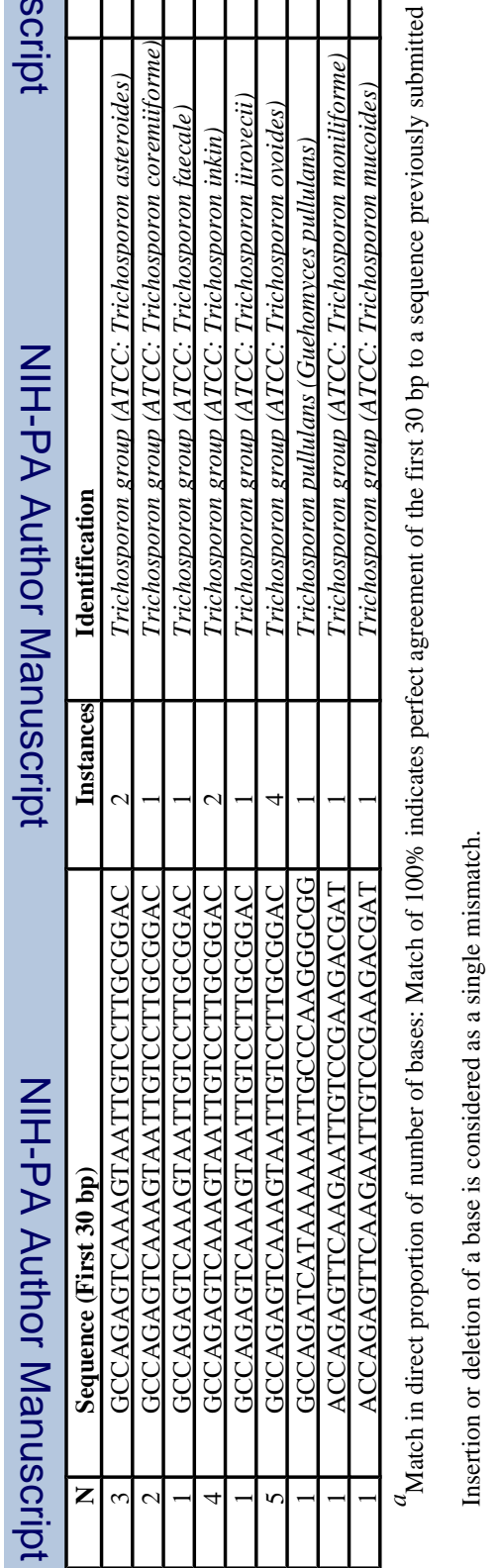

Eur J Clin Microbiol Infect Dis. Author manuscript; available in PMC 2009 September 1. 